\title{
Editorial Comment on: Radiation induced breast cancer risk in BRCA mutation carriers from low-dose radiological exposures: a systematic review Radioprotection 52(4), 231-240, DOI: 10.1051/radiopro/2017034
}

\author{
M. Bourguignon (Editor in Chief Radioprotection) ${ }^{*}$ \\ IRSN, BP17, 92262 Fontenay-aux-Roses, France.
}

In this issue of Radioprotection, C. Colin et al. present a systematic review of literature regarding the risk of breast cancer $(\mathrm{BC})$ related to low dose medical exposures to ionizing radiation in women carrying a BRCA mutation. This review synthesizes knowledge from different disciplines and approaches over the last two decades. This paper addresses a radiological protection issue of major public health interest straight in the editorial line of this journal (Bourguignon et al., 2017).

Increasing numbers of new $\mathrm{BC}$ cases and deaths are observed in all developed countries with a high psychosocial impact, only partially attributable to the increasing population age. In the European Union, there were 361608 new BC cases in 2012 with an increase to 373733 in $2015(+3.4 \%)$; deaths were 91585 and 95357 , respectively (+4.1\%) (IARC, 2016). In the general population, there are numerous reasons of the rather low societal acceptance of mammographic screening of $\mathrm{BC}$ programs. Among them, over diagnosis, false-positive consequences and the deleterious effects of ionizing radiation are the main reported concerns (Sardanelli et al., 2017).

BRCA genes are tumor-suppressor genes which proteins are deeply involved in the DNA damage response. Since a cancer process includes a number of persistent DNA damages (Hanahan and Weinberg, 2011), a genetic alteration of the DNA damage response in the BRCA carriers may constitute an increased radio-induced $\mathrm{BC}$ risk. Women with BRCA mutations have a very significant risk of spontaneous $\mathrm{BC}$ at a young age, reaching about $50 \%$ at age 50 and $70 \%$ at age 70 . Thus, BRCA1/2 mutation carriers are recommended to start MRI and also mammographic BC screening at age as young as 25-30 years. There is a major responsibility for the scientific and medical community considering the potential consequences of such X-ray exposures.

The merit of the review of Colin and colleagues (Colin et al., 2017) is to positively contribute to the debate by exploring in depth the data regarding BRCA mutation carriers. A systematic search was conducted addressing the main

\footnotetext{
*Corresponding author: michel. bourguignon@irsn.fr
}

question: how and why radiation exposure may affect $\mathrm{BC}$ risk with such low-doses? When risk modelling suggests an increased risk from X-ray low-dose exposures before age 40, most of the cohort data indicate an increased risk of radioinduced breast cancer in BRCA carriers less than 30 years with no current available data to investigate the risk of exposures after age 30 . These results clearly justify the conclusion that repeated breast exposures to ionizing radiation should be used very cautiously in BRCA carriers, especially when they are young. The best compromise needs to be found on the question: how to properly screen BC in BRCA carriers early enough without increasing the already high spontaneous risk of $\mathrm{BC}$ with exposures to ionizing radiation? For the first time in the world, the necessity to decrease screening breast X-ray exposures is reported in the new French guidelines for BC screening in BRCA1/2 mutations carriers by recommending only one systematic mammographic view per breast in association with MRI (INCa, 2017). A challenge for the radiologists will be to evaluate with accuracy the benefit/risk balance of mammography and MRI and the screening efficiency of new standardized breast ultrasound modalities (automated 3D ultrasound).

This review reported some responses about the epidemiological level of radio-induced $\mathrm{BC}$ risk in BRCA carriers and cellular consequences of exposures to ionizing radiation. By showing an increase risk in BRCA mutation carriers, it obviously contributes to the evidence of a high radiosusceptibility in this sub-population. This abnormal individual response to ionizing radiation (Foray et al., 2016) even at low dose has been identified as a priority in radiological protection (Bourguignon et al., 2017).

This raises a difficult dilemma: since BRCA carriers are at increased spontaneous risk of $\mathrm{BC}$ it is legitimate to strongly screen for it on the one hand, but is it legitimate to do it with ionizing radiation (mammography) which are known to be clastogenic even at low dose in such patients whose DNA damage response is altered?

The pioneer work of C. Colin and research team in her $\mathrm{PhD}$ thesis (Colin et al., 2011) clearly shows that some abnormal 
DNA damage response from high familial $\mathrm{BC}$ risk women and BRCA carriers can be clearly observed in human non-tumoral mammary epithelial cells exposed to low dose ionizing radiation in the exact condition of mammography. Interestingly, it is the only dedicated article in radiation biology which has been retrieved from literature in the review object of this editorial comment. By itself the abnormal DNA damage response of the BRCA carriers invites to a cautious use of ionizing radiation in these women who are already cancer prone. This justification is reinforced by epidemiological data.

The radiation biology work of Colin et al. mostly included women with high family risk of $\mathrm{BC}$ who were not BRCA carriers. The same observations of spontaneous and abnormal DNA damage response were made. A low dose effect, a supraadditive low and repeated dose effect (LORD), and a delayed additional and induced effect (LADI) were shown, the later being the sign of increased genomic instability. All together these results are in favor of the extended conclusions of Colin et al. (2017) to all patients with a family risk of BC in whom repeated breast exposures to ionizing radiation should be used very cautiously.

The abnormal DNA damage response pathways observed in BRCA carriers and in women with high family risk of BC should be thoroughly investigated since they may offer ways to screen for cancer prone patients who are also radio-susceptible to cancer. The functional proximity of BRCA proteins and of ATM is in favor of the potential usefulness of nuclear pATM assays developed for individual radiosensitivity screening (Granzotto et al., 2016; Ferlazzo et al., 2017) to individual radio-susceptibility to cancer screening.

Furthermore the DNA damage response pathways being at the crossroads of DNA damage signalization and repair, an abnormal DNA damage response is likely to be observed whatever the genetoxic insults. Consequently patients with BRCA mutations may be more susceptible to all genotoxic compounds. Should this hypothesis be confirmed not only exposures to low ionizing radiation dose should be justified in BRCA carriers but also exposures to all genotoxic compounds, even at low doses.

To sum up and to add responses to the crucial question of the mammographic screening risk in such women, some specific effects of low doses such as mammography exposures can be easily observed at the cellular level. Epidemiology reported that $\mathrm{BC}$ can be favored by very low cumulative $\mathrm{X}$-ray doses in BRCA carriers especially if those exposures start before age 30 , with no current available data on the risk of exposures after age 30 .

In conclusion, the review article of Colin et al. provides a scientific comprehensive update on the issue of radiosusceptibility to BC induction in BRCA carriers, and also opens the issue of the combination of effects of low dose of genetoxic compounds in cancer prone individuals.

\section{References}

Bourguignon M, Bérard P, Bertho JM, Farah J, Mercat C. 2017. What's next in Radioprotection?, Radioprotection 52: 21-28.

Colin C et al. 2011. DNA double-strand breaks induced by mammographic screening procedures in human mammary epithelial cells, Int. J. Radiat. Biol. 87: 1103-1112.

Colin C, Foray N, Di Leo G, Sardanelli F. 2017. Radiation induced breast cancer risk in BRCA mutation carriers from low-dose radiological exposures: a systematic review, Radioprotection 52(4): 231-240.

Ferlazzo M, Bourguignon M, Foray N. 2017. Functional assays for individula radiosensitivity: a critical review, Semin. Radiat. Oncol. 27: $310-315$.

Foray N, Bourguignon M, Hamada N. 2016. Individual response to ionizing radiation, Mutat. Res. 770: 369-386.

Granzotto A et al. 2016. Influence of nucleoshuttling of the ATM protein in the healthy tissues response to radiation therapy: toward amolecular classification of human radiosensitivity, Int. J. Radiat. Oncol. Biol. Phys. 94: 450-460.

Hanahan D, Weinberg RA. 2011. Hallmarks of cancer: the next generation, Cell 144: 646-674.

IARC. 2016. Globocan Online Analysis: Estimated cancer incidence, mortality and prevalence worldwide in 2012. Available from http://globocan.iarc.fr/Pages/burden_sel.aspx.

INCa. 2017. Thésaurus - Femmes porteuses d'une mutation de BRCA1 ou BRCA2 / Détection précoce du cancer du sein et des annexes et stratégies de réduction du risque. Collections/ Recommandations-et-referentiels. Available from http://www.ecancer.fr/Expertises-et-publications/Catalogue-des-publications/ Collections/Recommandations-et-referentiels.

Sardanelli F et al. 2017. Position paper on screening for breast cancer by the European Society of Breast Imaging (EUSOBI) and 30 national breast radiology bodies, Eur. Radiol. 27(7): 2737-2743.

Cite this article as: Bourguignon M. 2017. Editorial Comment on: Radiation induced breast cancer risk in BRCA mutation carriers from low-dose radiological exposures: a systematic review. Radioprotection 52(4): 229-230 